\title{
Breslow thickness of cutaneous malignant melanoma in paraffin wax and frozen sections
}

\author{
N Kirkham, K Blessing, B Gibson, M L Price
}

\begin{abstract}
Breslow tumour thickness was measured in frozen and paraffin wax sections from 21 excision biopsies of cutaneous malignant melanomas by two observers. There was no consistent variation between frozen and parafin wax sections, with recorded differences ranging from $+0.3 \mathrm{~mm}$ to $-0.2 \mathrm{~mm}$. Interobserver differences ranged from $+0.4 \mathrm{~mm}$ to $-0.2 \mathrm{~mm}$. The interobserver variations exceeded the intraobserver variations, but neither were significant. These findings show conclusively that, when using high quality frozen sections, there is no significant difference between Breslow thickness measured in frozen or paraffin wax sections and therefore that frozen sections can be used to microstage melanoma. Interobserver variations seem to be a more likely source of erroneous measurements of tumour thickness.
\end{abstract}

An important advance was made in our understanding of the behaviour of malignant melanoma when Clark introduced the concept of levels of invasion and related this to prognosis. ${ }^{1}$ This was further refined when Breslow described a method of measuring the thickness of the tumour in millimetres and showed a good correlation with clinical outcome. ${ }^{2}$ Breslow went on to comment on the relative ease with which the measurements can be made, the importance of adequate sampling of a tumour, because of variations in thickness from place to place, and the difficulties of defining the deepest malignant cell in a thick tumour. ${ }^{3}$ Breslow thickness is now widely regarded as the most important prognostic indicator, and surgical margins of excision are based on this measurement. ${ }^{45}$

In the immunohistochemical study of melanoma there are several markers which do not work in paraffin wax sections, so it is advantageous to be able to examine all or part of a biopsy specimen in frozen sections, without sacrificing any diagnostically useful feature of the specimen. We have shown that it is possible to cut high quality frozen sections of these specimens, which are suitable for morphological diagnosis. ${ }^{67}$ Whenever possible, of course, this should be done in conjunction with paraffin wax sections prepared from other parts of the same biopsy specimen. Indeed it is worth stating that the diagnosis of melanoma should not be made rashly, and that diagnosis made on frozen sections taken during excision biopsy has no part to play in routine histopathological practice.

One problem that has arisen in measuring tumour thickness is an alleged difference in tumour thickness between frozen and paraffin wax sections from the same lesion, which would make frozen sections unsuitable for microstaging. ${ }^{89}$ In this paper we address this question and report the results of a comparative study of our material.

\section{Methods}

Excision biopsy specimens of 21 pigmented lesions, with a clinical diagnosis of malignant melanoma, were sent fresh from the dermatology outpatients clinic to the histopathology laboratory. The biopsy specimens arrived within two hours of excision and were then sliced transversely through the centre of the lesion, using a fresh scalpel blade, to produce a block 1-2 mm thick, which was then placed in a capsule and stored in a liquid nitrogen specimen store, before the frozen sections were cut. ${ }^{10}$

The outer parts of the skin ellipses were then fixed in neutral, buffered, formol saline overnight, before blocks were taken off the cut ends of each half of the fixed biopsy specimen, for routine processing to produce haematoxylin and eosin stained paraffin wax sections.

For sectioning, the frozen blocks were orientated on the microtome chuck so that the line of cutting was at an angle of about $30^{\circ}$ to the line of the epidermal surface. Sections were cut along this line, at a thickness of $10 \mu \mathrm{m}$ and at a chuck temperature of $-40^{\circ} \mathrm{C}$, using a wedge profile knife and were stained with haematoxylin and eosin as previously described. ${ }^{6}$ The tissue blocks for paraffin wax sectioning were orientated so that the epidermis was parallel to the knife edge and was the first side of the block to be cut.

The frozen and paraffin wax sections from each biopsy specimen were reviewed and measured independently by two observers (NK and KB) to confirm the diagnosis of melanoma and to establish the Breslow thickness in the paired sections. Microstaging was performed using the Vernier method ${ }^{11}$ and confirmed using an ocular micrometer.

Statistical analysis was performed, using the paired $t$ test, to check for significant differences between measurements in frozen and paraffin wax sections in each biopsy specimen, and for differences in Breslow tumour depths as recorded on each biopsy specimen by the two observers. 

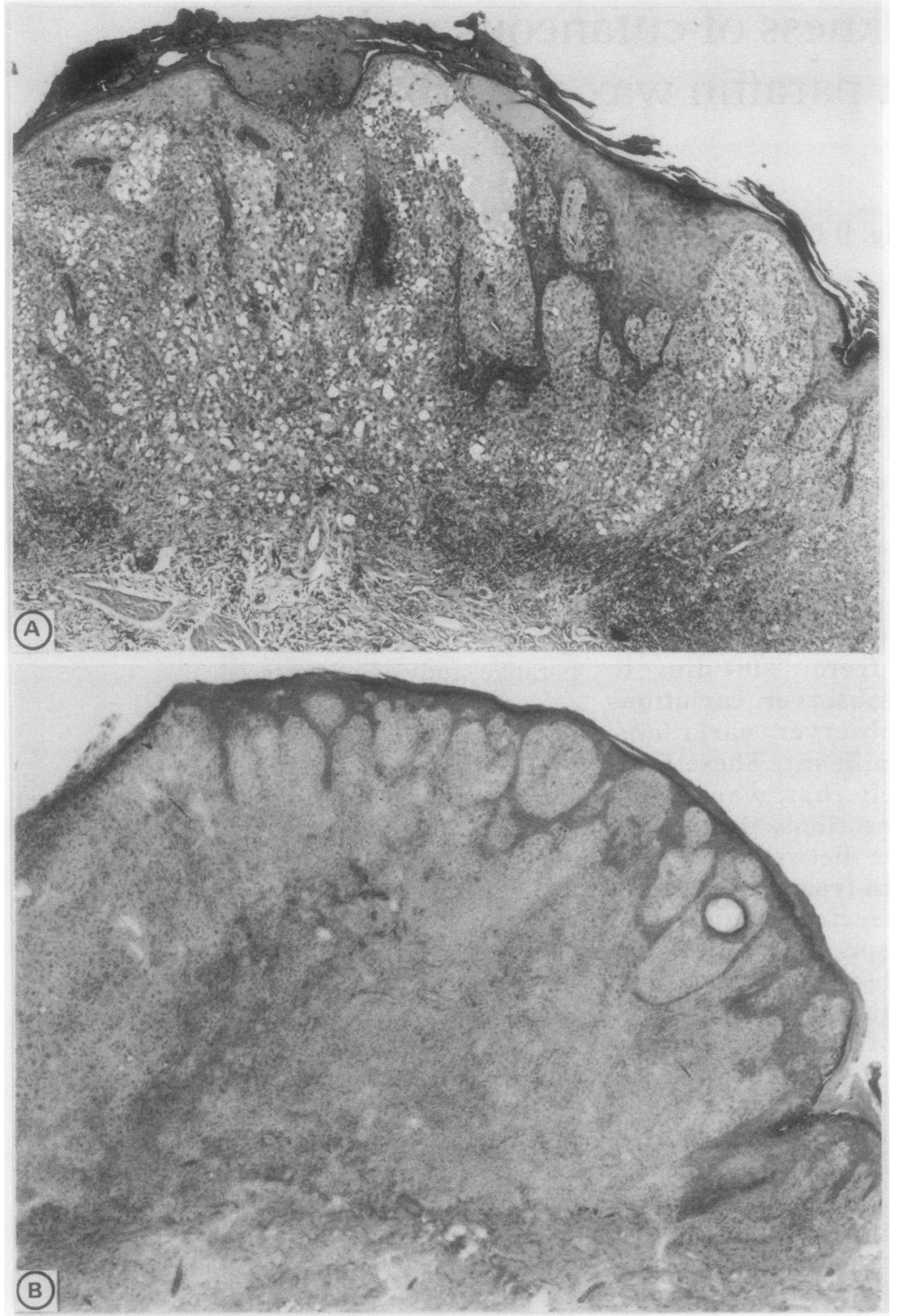

Malignant melanoma which was received fresh and bisected to produce $(A)$ paraffin wax and $(B)$ frozen sections of identical Breslow thickness (haematoxylin and eosin).

\section{Results}

The Breslow measurements showed a close agreement between frozen and paraffin wax sections for each biopsy specimen. There were no significant differences for either intraobserver or interobserver variations (table 1). The measurements were only made to one decimal place and so differences of $0.1 \mathrm{~mm}$ were difficult to avoid because of the effects of rounding. There was no significant correlation between the extent of the differences and the size of the tumour. The interobserver differences are, if anything, greater than the intraobserver differences (tables 2 and 3 ).

Other minor differences between frozen and paraffin wax section measurements may be attributable to variations within the tumours which made it difficult to produce exactly similar appearances in the adjacent blocks used for the sections. Twenty of the tumours were classified as malignant melanoma with an adjacent lateral component of superficial spreading type. The thickest tumour was the only nodular melanoma in the group.

\section{Discussion}

Previous comparative studies of Breslow tumour thickness in paraffin wax and frozen sections have shown conflicting results. Shafir $e t$ al concluded from their study that the difference in measurement between frozen and paraffin wax sections was never greater than $0.1 \mathrm{~mm}$, and also that the frozen sections were consistently thicker by $0.1 \mathrm{~mm}$. They advised that this should be taken into account when measuring thin tumours. ${ }^{8}$ We have not found a consistent difference between the two preparations from the same biopsy specimen.

In a similar study Nield et al found no constant relation between frozen and paraffin wax sections. ${ }^{9}$ They reported a variation of between $0.8 \mathrm{~mm}$ and $3.1 \mathrm{~mm}$, and recommended that clinical decisions should not be based on results obtained from frozen section.

Table 1 Breslow tumour thickness ( $\mathrm{mm}$ ) in frozen $(F)$ and paraffin $(P)$ sections by two observers $(A$ and $B)$, together with differences between measurements for section type and observer

\begin{tabular}{|c|c|c|c|c|c|c|}
\hline \multirow[b]{2}{*}{ Biopsy specimen No } & \multicolumn{2}{|c|}{ Frozen section } & \multicolumn{2}{|c|}{ Paraffin wax section } & \multicolumn{2}{|c|}{ Greatest difference } \\
\hline & $A$ & $B$ & $A$ & $B$ & $F: P$ & $A: B$ \\
\hline $\begin{array}{r}1 \\
2 \\
3 \\
4 \\
5 \\
6 \\
7 \\
8 \\
9 \\
10 \\
11 \\
12 \\
13 \\
14 \\
15 \\
16 \\
17 \\
18 \\
19 \\
20 \\
21\end{array}$ & $\begin{array}{l}0.2 \\
0.3 \\
0.4 \\
0.4 \\
0.5 \\
0.6 \\
0.6 \\
0.7 \\
0.7 \\
0.7 \\
0.8 \\
0.8 \\
0.8 \\
1.1 \\
1.2 \\
2.1 \\
2.4 \\
2.6 \\
2.7 \\
3.1 \\
7.5\end{array}$ & $\begin{array}{l}0.2 \\
0.4 \\
0.5 \\
0.4 \\
0.5 \\
0.4 \\
0.7 \\
0.7 \\
0.7 \\
0.7 \\
1.0 \\
0.8 \\
0.7 \\
1.3 \\
1.0 \\
2.2 \\
2.8 \\
2.7 \\
2.8 \\
3.2 \\
7.8\end{array}$ & $\begin{array}{l}0.2 \\
0.3 \\
0.4 \\
0.6 \\
0.6 \\
0.7 \\
0.7 \\
0.7 \\
0.7 \\
0.6 \\
0.7 \\
0.7 \\
0.8 \\
1.0 \\
1.3 \\
2.2 \\
2.4 \\
2.5 \\
2.6 \\
3.1 \\
7.6\end{array}$ & $\begin{array}{l}0.3 \\
0.5 \\
0.5 \\
0.6 \\
0.5 \\
0.5 \\
0.7 \\
0.7 \\
0.6 \\
0.6 \\
0.7 \\
0.7 \\
0.7 \\
1.3 \\
1.2 \\
2.4 \\
2.6 \\
2.7 \\
2.6 \\
3.0 \\
8.0\end{array}$ & $\begin{array}{l}0 \cdot 1 \\
0 \cdot 1 \\
0 \cdot 0 \\
0 \cdot 2 \\
0 \cdot 1 \\
0 \cdot 1 \\
0 \cdot 1 \\
0 \cdot 0 \\
0 \cdot 1 \\
0 \cdot 1 \\
0 \cdot 3 \\
0 \cdot 1 \\
0 \cdot 1 \\
0 \cdot 1 \\
0 \cdot 2 \\
0 \cdot 2 \\
0 \cdot 2 \\
0 \cdot 1 \\
0 \cdot 2 \\
0 \cdot 2 \\
0 \cdot 2\end{array}$ & $\begin{array}{l}0.1 \\
0.2 \\
0 \cdot 1 \\
0.0 \\
0 \cdot 1 \\
0.2 \\
0 \cdot 1 \\
0.0 \\
0 \cdot 1 \\
0.0 \\
0.2 \\
0.0 \\
0.1 \\
0.2 \\
0.2 \\
0.2 \\
0.4 \\
0.2 \\
0.1 \\
0.1 \\
0.4\end{array}$ \\
\hline
\end{tabular}

Paired $t$ test.

Frozen sections, observer $A: B, t=1.76 ; p=0.093$.

Paraffin wax sections, observer A:B, $t=1 \cdot 23 ; \mathrm{p}=0.23$

Frozen (mean of 2 observations); paraffin wax $t=0.1 ; \mathrm{p}=0.92$. 
Table 2 Intraobserver variation of Breslow tumour thickness $(\mathrm{mm})$ in frozen $(F)$ and paraffin wax $(P)$ sections (mean difference in parentheses)

\begin{tabular}{lll}
\hline Section type & $A$ & $B$ \\
\hline $\mathrm{F}=\mathrm{P}$ & 9 & 7 \\
$\mathrm{~F}>\mathrm{P}$ & $6(0 \cdot 10)$ & $6(0 \cdot 18)$ \\
$\mathrm{F}<\mathrm{P}$ & $7(0 \cdot 11)$ & $9(0 \cdot 11)$ \\
\hline
\end{tabular}

Table 3 Interobserver variation of Breslow tumour thickness $(\mathrm{mm})$ in frozen and paraffin wax sections for observers $A$ and $B$ (mean difference in parentheses)

\begin{tabular}{lcl}
\hline Observer & Frozen section & Paraffin wax section \\
\hline $\mathrm{A}=\mathrm{B}$ & 7 & 7 \\
$\mathrm{~A}>\mathrm{B}$ & $11(0 \cdot 13)$ & $8(0 \cdot 20)$ \\
$\mathrm{A}<\mathrm{B}$ & $4(0 \cdot 15)$ & $7(0 \cdot 11)$ \\
\hline
\end{tabular}

Their data, however, show larger discrepancies for thick melanomas. There was very good correlation within the thin group of tumours $(<1.0 \mathrm{~mm})$, where a difference in Breslow thickness may result in different surgical margins of excision. ${ }^{5}$ The difference is less crucial in thicker tumours. We also found less difference between observers in thinner tumours.

Obviously there are difficulties in obtaining sections of consistently high quality, but these problems apply almost equally to both paraffin wax and frozen sections. The angle of cutting, the section thickness, the cutting temperature, and the sharpness of the cutting edge can all play a part in producing variations in tumour thickness in the final section. When attention is given to specimen sampling, however, and section cutting is performed with a high level of technical expertise, then sections of adequate quality for diagnosis and measurement can be obtained. It is impossible to avoid some genuine differences in tumour thickness, when the frozen paraffin wax sections are cut from different blocks of tissue taken from different parts of the tumour. Nevertheless, in this study such differences seemed to be less than the statistically insignificant interobserver differences.

It is perhaps more important to pay attention to the nature of the observer rather than the observation. This study used two observers who are experienced in the diagnosis of melanoma, ${ }^{61112}$ and even though there was no significant difference between the various pairs of measurements, absolute agreement could still not be achieved. It has been suggested that interobserver variation may be so great that it would invalidate the measurement. ${ }^{13}$ Breslow showed how training of his residents improved their congruence in measuring melanomas, ${ }^{3}$ so it is important that pathologists who are to measure melanomas should be adequately trained. The Vernier method is the most accurate method of measurement in routine practice. ${ }^{91314}$

In conclusion, irrespective of the type of section used, the Breslow measurement of a melanoma should be made by a trained observer and should only be made if it is clear that the whole tumour has been adequately sampled, so as to identify the maximum thickness. There is no significant difference between Breslow tumour thickness in either frozen or paraffin wax sections of melanoma.

We thank Dr Peter Cheek, Brighton Polytechnic, for statistical advice.

1 Clark WH Jr, From L, Bernardino EA, Mihn MC. The histogenesis and biological behaviour of primary malig nant melanoma. Cancer Res 1969;29:705-27.

2 Breslow A. Thickness, cross sectional area and depth of invasion in the prognosis of cutaneous melanoma. Ann Surg 1970;172:902-7.

3 Breslow A. Problems in the measurement of tumour thickness and level of invasion in cutaneous melanoma. Hum Pathol 1977;8:1-2.

4 Balch CM, Murad TM, Soong S, Griffin AL, Halpern NB, Maddox WA. A multifactorial analysis of melanoma: 1 . Prognostic histopathological features comparing Clark's and Breslow's staging methods. Ann Surg 1978;188: and Brestor.

5 Veronesi $\mathrm{U}$, Cascinelli $\mathrm{N}$, Adamus $\mathrm{J}$, et al. Thin stage 1 primary cutaneous malignant melanoma. Comparison of excision with margins of 1 or $3 \mathrm{~cm}$. N Engl J Med 1988; 318:1159-62.

6 Kirkham N, Price ML, Gibson B, Leigh IM, Coburn P, Darley C. Type VII Collagen antibody LH $7 \cdot 2$ identifies basement membrane characteristics of thin malignant melanomas. J Pathol 1989;157:243-7.

7 Kirkham N, Gibson B, Leigh IM, Price ML. A comparison of antibodies to type VII and type IV collagen laminin and amnion as epidermal basement membrane markers. $J$ Pathol 1989;159:5-6.

8 Shafir R, Hiss J, Tsur H, Bubis JJ. Pitfalls in frozen section diagnosis of malignant melanoma. Cancer 1983;51: $1168-70$

9 Nield DV, Saad MN, Khoo CT, Ali MH. Tumour thickness in malignant melanoma: the limitations of frozen section Br J Plast Surg 1988;41:403-7.

10 McGovern VJ, Cochran AJ, Van der Esch EP, Little JH, MacLennan $R$. The classification of malignant melanoma, its histological reporting and registration: a revision of the 1972 Sydney Classification. Pathology 1986;18:12-21.

11 Kirkham N, Cotton DW. Measuring melanomas; the Vernier method. J Clin Pathol 1984;37:229-30.

12 Blessing K, McLaren KM, McLean A, Davidson P. Thin malignant melanomas $(<1.5 \mathrm{~mm})$ with metastasis: a histological study and survival analysis. Histopathol 1990;17:389-96.

13 Colloby PS, West KP, Fletcher A. Clinical implication of observer variations in malignant melanoma measurements. J Pathol 1990;161:350A.

14 Calder CJ, Campbell AP, Plastow SR. Measurement techniques for melanoma: a statistical comparison. J Clin Pathol 1990;43:922-3. 